\title{
An Investigation of Water Diffusivity Changes along the Perivascular Space in Elderly Subjects with Hypertension
}

\author{
(D). Kikuta, (D). Kamagata, (D). Takabayashi, (D). Taoka, (D) H. Yokota, (D). Andica, (D) A. Wada, (D). Someya, (D). Tamura,
}

(D) Kawamori, (D) H. Watada, (D)S. Naganawa, and (D). Aoki

\section{ABSTRACT}

BACKGROUND AND PURPOSE: Hypertension may be related to alterations of the glymphatic system, a waste metabolite drainage system in the brain. We aimed to investigate analysis along the perivascular space index changes in elderly subjects with hypertension.

MATERIALS AND METHODS: Diffusion-weighted images were acquired from 126 subjects, including 63 subjects with hypertension (25 men and 38 women; mean age, 72.45 years) and 63 age- and sex-matched controls (25 men and 38 women; mean age, 72.16 years). We calculated the analysis along the perivascular space index as a ratio of the mean of $x$-axis diffusivities in the projection and association areas to the mean of $y$-axis diffusivity in the projection area and z-axis diffusivity in the association area. The left, right, and mean analysis along the perivascular space indices of both hemispheres were compared between the hypertension and control groups using a Mann-Whitney $U$ test. The Spearman correlation coefficient was used to assess the correlation between the left, right, and mean ALPS indices and blood pressure and pulse pressure.

RESULTS: The left $(P=.011)$ and mean $(P=.024)$ analysis along the perivascular space indices of the hypertension group were significantly lower than that of the control group. The left, right, and mean analysis along the perivascular space indices of all subjects were significantly negatively correlated with blood pressure values $(r=-0.200$ to $-0.278, P=.002-0.046)$ and pulse pressure values $(r=-0.221$ to $-0.245, P=.006-0.013)$.

CONCLUSIONS: Our results are consistent with a model in which hypertension causes glymphatic dysfunction.

ABBREVIATIONS: ALPS = analysis along the perivascular space; $\mathrm{HT}=$ hypertension; ISF = interstitial fluid

$\mathbf{T}$ he glymphatic system is a waste metabolite drainage system in the brain. ${ }^{1-3}$ It consists of four sequential anatomic segments. First, the cerebrospinal fluid (CSF) flows along the perivascular space surrounding the penetrating arteries. Second, the CSF disperses into the interstitial space and transfers waste metabolites into the interstitial fluid (ISF) facilitated by

Received April 6, 2021; accepted after revision September 6

From the Department of Radiology (J.K., K.K., K.T., C.A., A.W., S.A.), Sportology Center (Y.S., Y.T., R.K., H.W.), and Department of Metabolism \& Endocrinology (Y.T., R.K., H.W.), Juntendo University Graduate School of Medicine, Tokyo, Japan; Department of Innovative Biomedical Visualization (T.T.), Graduate School of Medicine, Nagoya University, Aichi, Japan; Department of Diagnostic Radiology and Radiation Oncology (H.Y.), Graduate School of Medicine, Chiba University, Chiba, Japan; and Department of Radiology (S.N.), Nagoya University Graduate School of Medicine, Aichi, Japan.

This work was supported, in part, by the Japan Society for the Promotion of Science Grants-in-Aid for Scientific Research, grant Nos. 18H02772 and 20K16737, and a Grant-in-Aid for Special Research in Subsidies for ordinary expenses of private schools from the Juntendo Research Branding Project.

Please address correspondence to Junko Kikuta, MD, PhD, Department of Radiology, Juntendo, University of Medicine, 2-1-1 Hongo, Bunkyo-ku, Tokyo 1138421, Japan; e-mail: j.kikuta.hy@juntendo.ac.jp

- Indicates open access to non-subscribers at www.ajnr.org

http://dx.doi.org/10.3174/ajnr.A7334 aquaporin-4 water channels, which form the outer wall of the perivascular space. Third, the ISF-containing waste metabolites flow out of the large-caliber draining vein. ${ }^{2}$ Finally, interstitial solutes exit the brain through meningeal lymphatic vessels, together with the venous sinuses. ${ }^{4}$ Glymphatic dysfunction leads to the deposition of toxic waste products, such as amyloid $\beta$ and tau proteins, which contribute to the pathogenesis of Alzheimer disease. ${ }^{5-7}$ Toxic solute accumulation due to drainage dysfunction has been demonstrated in normal aging, ${ }^{8}$ traumatic brain injury, ${ }^{9}$ and stroke. ${ }^{10}$

Hypertension (HT) is a risk factor for Alzheimer disease in the elderly. ${ }^{11,12}$ HT involves pathologic alterations of small cerebral blood vessels and capillaries. ${ }^{13,14}$ Mestre et $\mathrm{al}^{13}$ found that the perivascular pump is less efficient in hypertensive mice due to dynamic changes in the vessel wall. Moreover, Mortensen et $\mathrm{al}^{14}$ demonstrated suppression of glymphatic activity in spontaneously hypertensive rats, which reduced parenchymal waste transport.

On the basis of these findings, we hypothesized that patients with HT have an impairment of the glymphatic system. Alterations in glymphatic function due to HT in living human brains have not been reported to date because of the challenges 
Table 1: Demographic characteristics of the participants ${ }^{a}$

\begin{tabular}{lccc}
\hline & $\begin{array}{c}\text { Control Group } \\
(\boldsymbol{n}=\mathbf{6 3})\end{array}$ & $\begin{array}{c}\text { HT Group } \\
(\boldsymbol{n}=\mathbf{6 3})\end{array}$ & $\boldsymbol{P}$ Values \\
\hline Age & $72.16(\mathrm{SD}, 5.11)$ & $72.45(\mathrm{SD}, 5.26)$ & 1 \\
Sex (male/female) & $25 / 38$ & $25 / 38$ & 1 \\
Systolic blood pressure $(\mathrm{mm} \mathrm{Hg})$ & $123.55(\mathrm{SD}, 10.09)$ & $143.63(\mathrm{SD}, 14.51)$ & $<.001$ \\
Diastolic blood pressure $(\mathrm{mm} \mathrm{Hg})$ & $80.30(\mathrm{SD}, 6.85)$ & $88.60(\mathrm{SD}, 9.30)$ & $<.001$ \\
Average blood pressure $(\mathrm{mm} \mathrm{Hg})$ & $98.86(\mathrm{SD}, 7.96)$ & $113.76(\mathrm{SD}, 11.37)$ & $<.001$ \\
Pulse pressure $(\mathrm{mm} \mathrm{Hg})$ & $67.56(\mathrm{SD}, 12.28)$ & $82.13(\mathrm{SD}, 13.86)$ & $<.001$ \\
Body mass index $\left(\mathrm{kg} / \mathrm{m}^{2}\right)$ & $21.56(\mathrm{SD}, 2.72)$ & $23.76(\mathrm{SD}, 2.55)$ & $<.001$ \\
Hemoglobin Alc $(\%)$ & $5.83(\mathrm{SD}, 0.67)$ & $5.76(\mathrm{SD}, 0.40)$ & .654 \\
Total cholesterol $(\mathrm{mg} / \mathrm{dL})$ & $216.73(\mathrm{SD}, 49.08)$ & $203.68(\mathrm{SD}, 33.92)$ & .093 \\
High-density lipoprotein $(\mathrm{mg} / \mathrm{dL})$ & $63.98(\mathrm{SD}, 13.31)$ & $64.89(\mathrm{SD}, 15.45)$ & .988 \\
Low-density lipoprotein $(\mathrm{mg} / \mathrm{dL})$ & $129.89(\mathrm{SD}, 31.54)$ & $119.33(\mathrm{SD}, 30.01)$ & .043 \\
Triglyceride $(\mathrm{mg} / \mathrm{dL})$ & $94.46(\mathrm{SD}, 65.70)$ & $97.17(\mathrm{SD}, 46.48)$ & .415 \\
Deep white matter hyperintensities & & & \\
$\quad$ Fazekas scale & $1.16(\mathrm{SD}, 0.48)$ & $1.41(\mathrm{SD}, 0.64)$ & .013 \\
\hline
\end{tabular}

${ }^{\mathrm{a}}$ Data are presented as means (SDs).

encountered during in vivo tracer studies. Taoka et $\mathrm{al}^{15}$ introduced the analysis along the perivascular space (ALPS) method, which is calculated using DWI as a noninvasive tool to evaluate the glymphatic system of living humans. This method assumes that diffusion plays an essential role in fluid transport in the brain parenchyma. We assessed ALPS index differences between elderly subjects with and without HT and evaluated the association between the ALPS index and blood pressure and pulse pressure.

\section{MATERIALS AND METHODS}

This study was approved by the ethics committee of Juntendo University in November 2015 and was conducted according to the principles outlined in the Declaration of Helsinki.

\section{Study Participants}

The Bunkyo Health Study is a prospective cohort study that has been running for $>10$ years. ${ }^{16}$ We recruited elderly subjects $65-$ 84 years of age who were living in Bunkyo-ku, an urban area in Tokyo. Our cohort comprised 1629 elderly people. Of these, 160 participants underwent both FLAIR imaging and DWI. All subjects were right-handed and had no history of diabetes (hemoglobin Alc $<6.5 \%$ ), hyperlipidemia (total cholesterol $<240 \mathrm{mg} / \mathrm{dL}$, low-density lipoprotein $<140 \mathrm{mg} / \mathrm{dL}$, high-density lipoprotein $>40 \mathrm{mg} / \mathrm{dL}$, and triglyceride $<150 \mathrm{mg} / \mathrm{dL}$ ), or obesity (body mass index $<25 \mathrm{~kg} / \mathrm{m}^{2}$; Table 1). Subjects with systolic/diastolic blood pressure of $\geq 135 / 85 \mathrm{~mm} \mathrm{Hg}$ during their first visit or those who had a history of using antihypertensive drugs were included in the HT group. We included 126 elderly participants, including 63 subjects with HT ( 25 men and 38 women; mean age, 72.45 [SD, 5.26] years) and 63 age- and sex-matched subjects without HT (controls; 25 men and 38 women; mean age, 72.16 [SD, 5.11] years) in this study. The demographic characteristics of the study subjects are presented in Table 1.

\section{MR Imaging Acquisition}

We performed FLAIR imaging $(\mathrm{TR}=11,000 \mathrm{~ms}$; $\mathrm{TE}=100 \mathrm{~ms}$; $\mathrm{TI}=2000 \mathrm{~ms}$; section thickness $=5 \mathrm{~mm}$ ) on a $3 \mathrm{~T} \mathrm{MR}$ imaging scanner (Airis Vento; Hitachi) in all participants. Deep white hyperintensity evaluation using the Fazekas scale based on axial FLAIR imaging was performed by an experienced neuroradiologist. DWI data were acquired on a $3 \mathrm{~T}$ MR imaging scanner (Magnetom Prisma; Siemens) with a 64-channel head coil. Echoplanar imaging was acquired using a b-value of $1000 \mathrm{~s} / \mathrm{mm}^{2}$ along 64 isotropic diffusion gradients in the anterior-posterior phase-encoding direction with the following parameters: $\mathrm{TR}=3300 \mathrm{~ms} ; \mathrm{TE}=70 \mathrm{~ms}$; FOV $=229 \times 229 \mathrm{~mm}$; matrix size $=$ $130 \times 130$; resolution $=1.8 \times 1.8 \mathrm{~mm}$; section thickness $=1.8 \mathrm{~mm}$; acquisition time $=7$ minutes 29 seconds. Each DWI acquisition was completed with a $b=0$ image. We also acquired standard and reverse phase-encoded blipped images with no diffusion weighting (blip-up and blipdown) to correct for magnetic susceptibility-induced distortions related to echo-planar imaging acquisitions.

\section{DWI Processing}

DWI data were processed using the FMRIB Software Library, ${ }^{17}$ Version 6.0 (FSL; www.fmrib.ox.ac.uk/fsl). Diffusion-weighted data were corrected for susceptibility-induced geometric distortions, eddy current distortions, and intervolume subject motion using the eddy and topup toolboxes. Diffusivity maps of each subject were acquired in the directions of the $\mathrm{x}$ - (right-left, $D x x$ ), $\mathrm{y}$ - (anterior-posterior, $D y y$ ), and z-axes (inferior-superior, $D z z){ }^{18}$ $D x x$ corresponds to the direction of the deep white matter vessels in the periventricular white matter. Considering that the glymphatic system runs along these deep white matter vessels, the Dxx is assumed to partly reflect water diffusivity along the glymphatic system. Fractional anisotropy maps of all study participants were also generated and registered to the FMRIB58_FA standard space (https://fsl.fmrib.ox.ac.uk/fsl/fslwiki/FMRIB58_FA) using FSL's Linear Image Registration Tool (FLIRT; http://www.fmrib.ox.ac. $\mathrm{uk} / \mathrm{fsl} /$ fslwiki/FLIRT) and nonlinear registration tool (FNIRT; http://fsl.fmrib.ox.ac.uk/fsl/fslwiki/FNIRT).

\section{ROI Placement}

For ROI placement, 1 subject (68-year-old female control participant) with minimal head movement (ie, with the smallest sum of squared difference) was selected. Using this subject's color-coded fractional anisotropy map, we manually placed 5$\mathrm{mm}$-diameter square ROIs in the projection and association areas at the level of the ventricle bodies of the left and right hemispheres (Fig 1). In the projection area, dominant fibers run in the $\mathrm{z}$-axis direction, perpendicular to both the $\mathrm{x}$ - and $\mathrm{y}$-axes, whereas in the association area, dominant fibers run in the $y$ axis direction, perpendicular to both the $\mathrm{x}$ - and $\mathrm{z}$-axes. The resulting ROIs were then registered to the same fractional anisotropy template. Finally, we manually checked the positions of the ROIs on each participant's fractional anisotropy image. Manual corrections were not performed because all ROIs were correctly placed. 


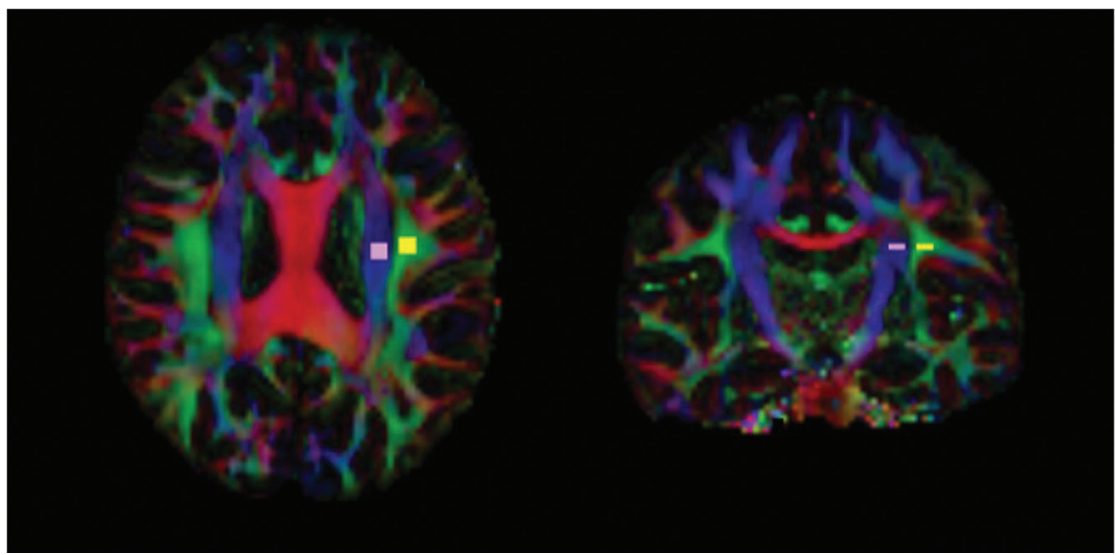

FIG 1. ROI placement for the calculation of the ALPS index. Square ROls of $5 \times 5 \mathrm{~mm}^{2}$ were placed in the projection (pink) and association (yellow) areas.

Table 2: The values of the left, right, and mean ALPS indices in the HT and control groups ${ }^{a}$

\begin{tabular}{lccc}
\hline & HT Group & Control Group & P Value \\
\hline Left ALPS index & $1.30(\mathrm{SD}, 0.22)$ & $1.40(\mathrm{SD}, 0.19)$ & .011 \\
Right ALPS index & $1.32(\mathrm{SD}, 0.18)$ & $1.37(\mathrm{SD}, 0.21)$ & .094 \\
Mean ALPS index & $1.31(\mathrm{SD}, 0.19)$ & $1.39(\mathrm{SD}, 0.19)$ & .024 \\
\hline
\end{tabular}

${ }^{a}$ Data are presented as means (SDs).

\section{ALPS Index Calculation}

The diffusivity values of the $\mathrm{x}^{-}, \mathrm{y}^{-}$, and $\mathrm{z}$-axes within the ROIs were obtained for each participant. The ALPS index was calculated as a ratio of the mean of the $\mathrm{x}$-axis diffusivity in the projection area (Dxxproj) and $\mathrm{x}$-axis diffusivity in the association area (Dxxassoc) to the mean of the $y$-axis diffusivity in the projection area (Dyyproj) and the $\mathrm{z}$-axis diffusivity in the association area (Dzzassoc) as follows:

$$
\text { ALPS index }=\frac{\text { Dxxproj }+ \text { Dxxassoc }}{\text { Dyyproj }+ \text { Dzzassoc }} \text {. }
$$

An ALPS index close to 1.0 reflects minimal diffusion along the perivascular space, whereas higher values indicate greater diffusivity. The left and right ALPS indices and the mean ALPS index of the left and right hemispheres were calculated.

\section{Statistical Analysis}

Statistical analysis was performed using SPSS Statistics, Version 27.0 (IBM). The left, right, and mean ALPS indices of the control and HT groups were compared using a nonparametric MannWhitney $U$ test. The associations between the left, right, and mean ALPS indices of all subjects and the values of systolic blood pressure, diastolic blood pressure, average blood pressure, and pulse pressure were evaluated using Spearman correlation coefficients. A $P$ value $<.05$ was considered statistically significant.

\section{RESULTS}

The features of the control and HT groups are summarized in Table 1. The control and HT groups did not differ significantly in age, sex, hemoglobin A1c, total cholesterol, high-density lipoprotein, or triglyceride values. As expected, the HT group had significantly higher $(P<.001)$ systolic, diastolic, and average blood pressures and pulse pressures than the control group. Furthermore, the HT group had significantly higher body mass index $(P<.001)$ and Fazekas scale scores $(P<.013)$ than the control group. In contrast, the low-density lipoprotein value of the control group was significantly higher than that of the HT group $(P<.043)$. Nevertheless, all subjects were without obesity or hyper-lowdensity lipoprotein cholesterolemia. Moreover, we confirmed no white matter hyperintensities in the ROIs.

The left $(P=.011)$ and mean ALPS $(P=.024)$ indices of the HT group were significantly lower than those of the control group. However, the right ALPS index did not differ significantly between the HT and control groups $(P=.094)$. Table 2 shows the left, right, and mean ALPS index values of the HT and control groups. Figure 2 shows the box plots of the left, right, and mean ALPS indices between the HT and control groups. Furthermore, the left, right, and mean ALPS indices of all subjects were significantly negatively correlated with systolic blood pressure values ( $r=-0.202$ to $-0.263, P=.003-.023$ ), diastolic blood pressure values $(r=-0.200$ to -0.218 , $P=.014-.046)$, average blood pressure values $(r=-0.231$ to $-0.278, P=.002-.009)$, and pulse pressure values $(r=-0.221$ to $-0.245, P=.006-.013)$. The scatterplots in Fig 3 show the correlation between the left, right, and mean ALPS indices and systolic blood pressure, diastolic blood pressure, average blood pressure, and pulse pressure values.

\section{DISCUSSION}

We evaluated elderly subjects with HT using the ALPS index, which is a potential biomarker for the assessment of glymphatic activity in the living human brain. Our results demonstrated that left and mean ALPS indices in the subjects with HT were significantly lower relative to controls, though no significant difference was observed between the HT and control groups for the right ALPS index. Our study also found negative correlations between the left, right, and mean ALPS indices and blood pressure, and the left, right, and mean ALPS indices and pulse pressure, which suggest that glymphatic dysfunction is related to HT.

HT increases the risk of cognitive impairment, vascular dementia, and Alzheimer disease in the elderly. ${ }^{11,12,19,20}$ The accumulation of toxic solutes such as amyloid $\beta$ and tau protein in the brain was shown to be an important neuropathologic mechanism of cognitive dysfunction in elderly subjects with HT. ${ }^{5-7}$ Previous studies have indicated that the accumulation of waste metabolites causes glymphatic dysfunction, which leads to further deposition. ${ }^{1-3} \mathrm{HT}$ and arteriosclerosis have been shown to lead to pathologic changes in small cerebral blood vessels and capillaries. ${ }^{13,14}$ Such abnormalities contribute to reductions in cerebral blood flow and volume, which cause white matter hypoperfusion. Moreover, endothelial cell abnormalities and blood-brain barrier dysfunction may contribute 

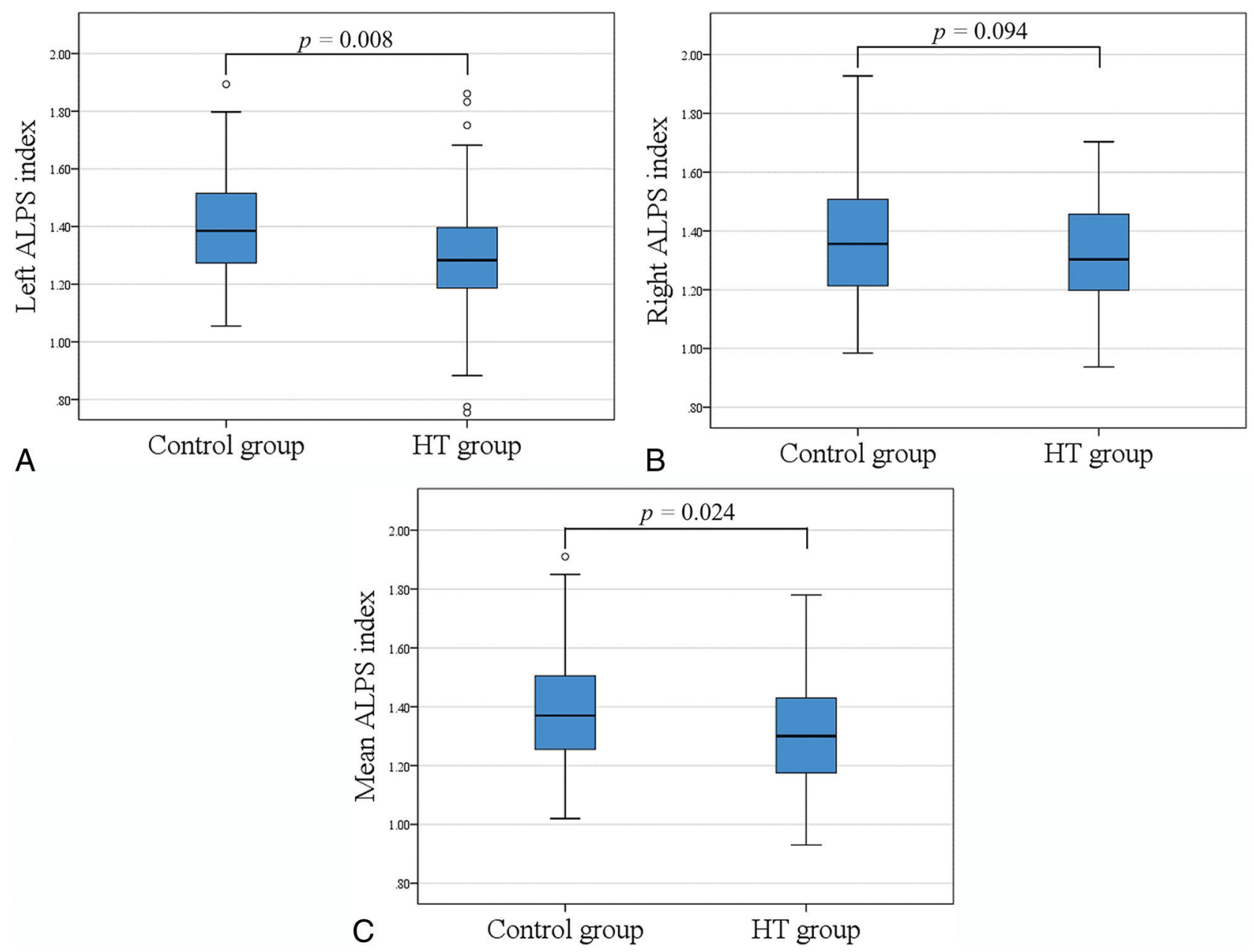

FIG 2. Boxplots of the left, right, and mean ALPS indices in the HT and control groups.

to white matter impairment. In the present study, although we did not detect any white matter hyperintensities in the ROIs, the HT group had more advanced white matter impairment than the control group. Adler et $\mathrm{al}^{21}$ demonstrated that blood-brain barrier disruption increases the permeability of the vessel wall and mobilizes inflammatory factors such as macrophages, lymphocytes, and complementary components, which may lead to myelin damage. Yamamoto et $\mathrm{al}^{22}$ also reported dilation of the perivascular space, accompanied by myelin degeneration and defects in ISF drainage in cerebral autosomal dominant arteriopathy, with subcortical infarcts and leukoencephalopathy associated with extensive smallvessel disease. In addition, Kamagata et $\mathrm{al}^{23}$ reported an association between arterial stiffness and white matter demyelination using magnetization transfer saturation imaging. Notably, myelin-rich tissue, such as white matter, has been shown to be more sensitive to ISF flow obstruction. ${ }^{24}$ Therefore, small-vessel diseases may be associated with alterations of both white matter microstructure and drainage function. Furthermore, in small-vessel disease, because of the difference in the anatomic bifurcation of the left and right carotid arteries, the left carotid artery is considered more susceptible to strong pulse pressure directly from the aortic arch, which increases the likelihood of plaque formations and intima damage becoming more severe. ${ }^{25}$ Thus, microangiopathy due to HT in the left cerebral hemisphere may be more marked than that in the right cerebral hemisphere. This possibility may explain why the ALPS indices of the left hemisphere showed a significant difference between the HT and control groups, which was not observed in the right hemisphere.

To the best of our knowledge, this is the first study to show changes in water diffusivity along the perivascular space in living patients with HT. Previously, the suppression of glymphatic function has been observed in spontaneously hypertensive rats using dynamic contrast-enhanced MR imaging, ${ }^{14}$ which suggested that hypertensive conditions reduce the effectiveness of arterial pulsation as a driver of CSF-ISF exchange and impair glymphatic activity. Using ex vivo fluorescence imaging, Mestre et $\mathrm{al}^{13}$ also demonstrated that the perivascular pump is less efficient in hypertensive mice due to changes in vessel wall dynamics. The perivascular pump driven by arterial pulsation is a powerful mechanism for fluid transport within the brain. ${ }^{26}$ Therefore, the decreased function of the perivascular pump could lead to a reduction in glymphatic activity. In line with previous studies in hypertensive animals, our findings demonstrated the effect of HT on human glymphatic dysfunction. Our study observed weak correlations between the ALPS index and blood pressure and the ALPS index and pulse pressure. Bewick et $\mathrm{al}^{27}$ reported that a weak correlation with a low $R$-value can be statistically significant in a large sample size. We used a relatively large sample size; thus, a weak correlation 

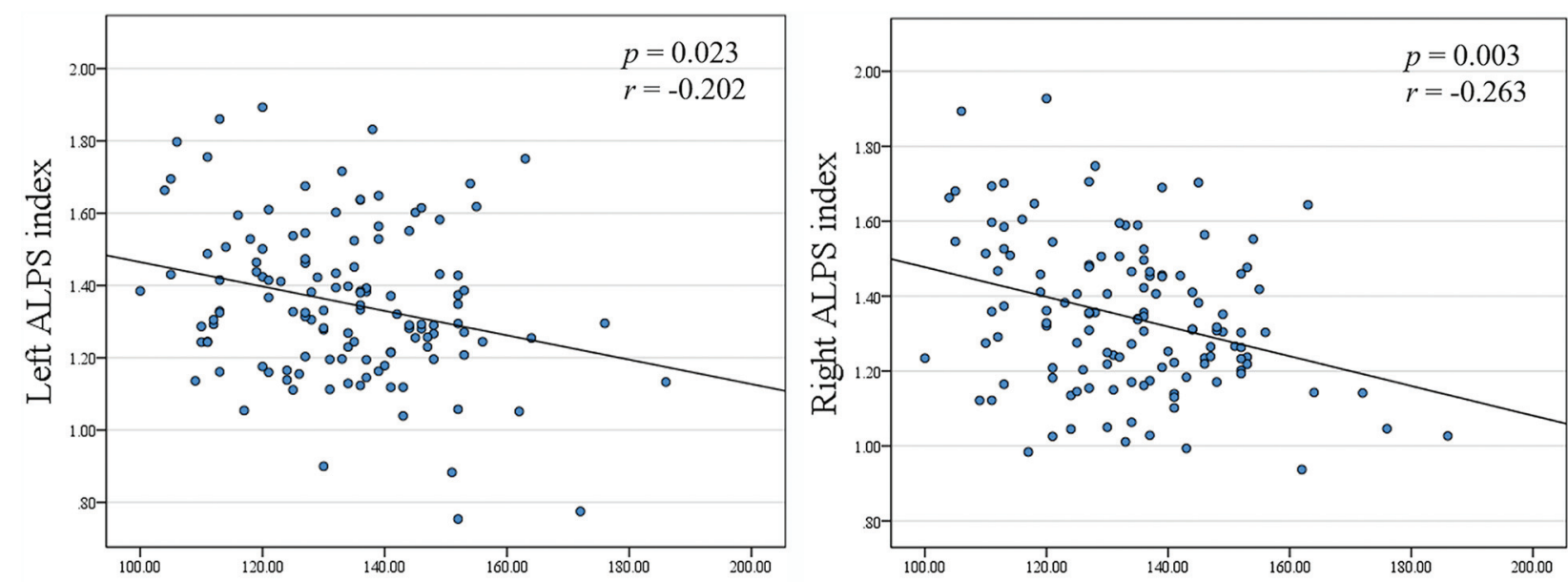

A Systolic blood pressure

B

Systolic blood pressure
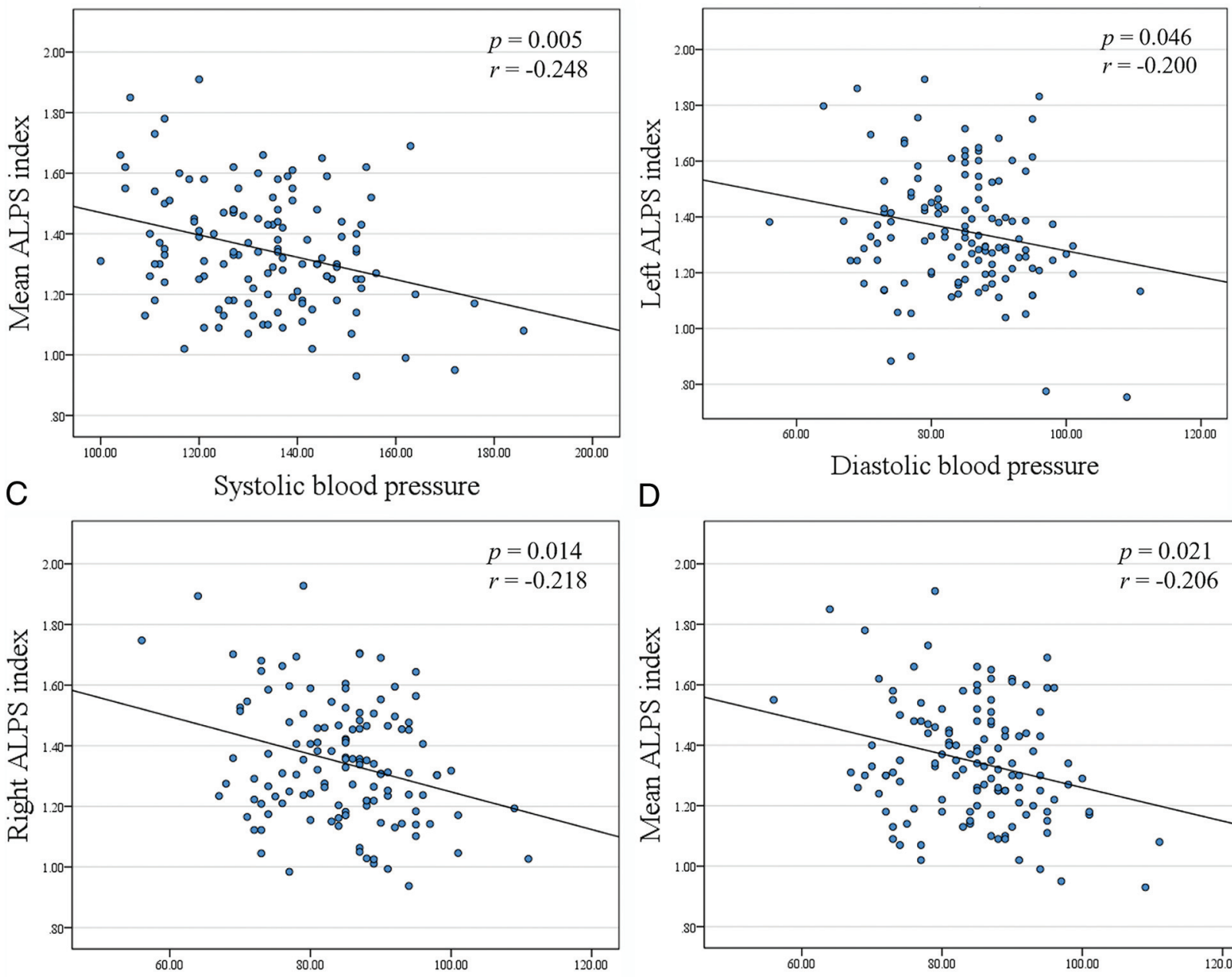

E

Diastolic blood pressure

$\mathrm{D}$

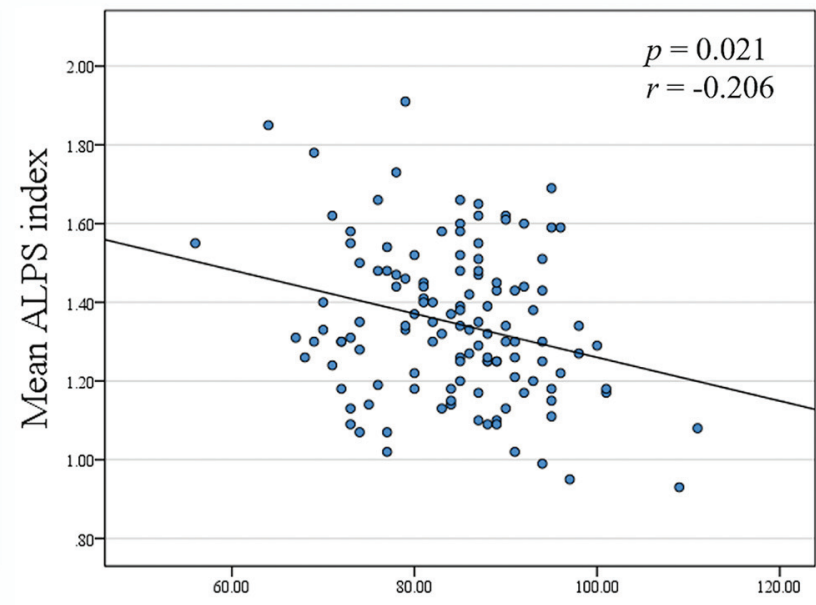

$\mathrm{F}$

Diastolic blood pressure

FIG 3. Scatterplots. A-C, Correlations between the left, right, and mean ALPS indices and the systolic blood pressure values. $D-F$, Correlations between the left, right, and mean ALPS indices and the diastolic blood pressure values. G-I, Correlations between the left, right, and mean ALPS indices and the average blood pressure values. $J-L$, Correlations between the left, right, and mean ALPS indices and the pulse pressure values in all subjects.

is still considered clinically relevant. High pulse pressure reflects an estimate of the stiffness of the large central arteries, and high blood pressure results from arteriosclerosis in the peripheral arteries. ${ }^{28}$ Such arterial damage may lead to glymphatic dysfunction due to arterial pulsation and perivascular pump changes. ${ }^{1,29,30}$
The clearance of waste metabolites is essential for tissue homeostasis and is mediated by the blood-brain barrier and the CSF-ISF exchange pathway. ${ }^{31}$ The glymphatic system is a clearance mechanism associated with CSF and ISF dynamics, as observed in many tracing studies. Iliff et $\mathrm{al}^{1}$ showed that CSF 

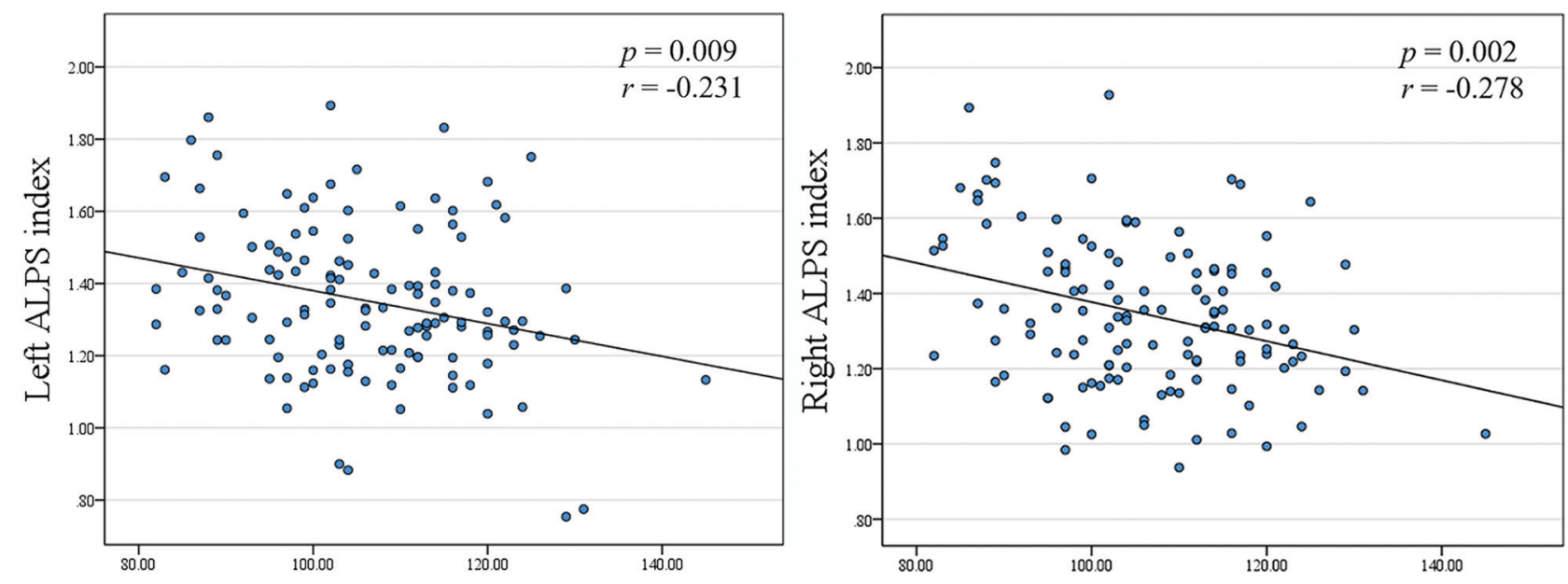

G Average blood pressure

$\mathrm{H}$

Average blood pressure
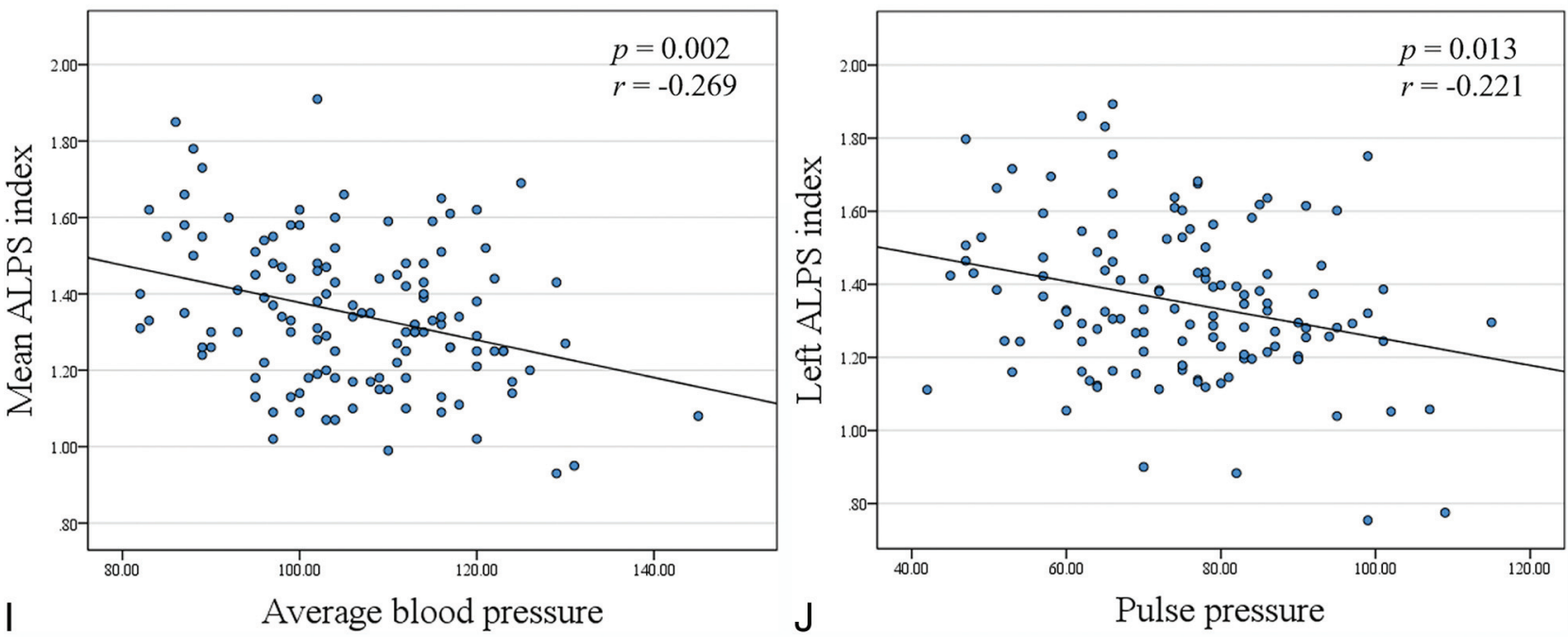

I

Average blood pressure
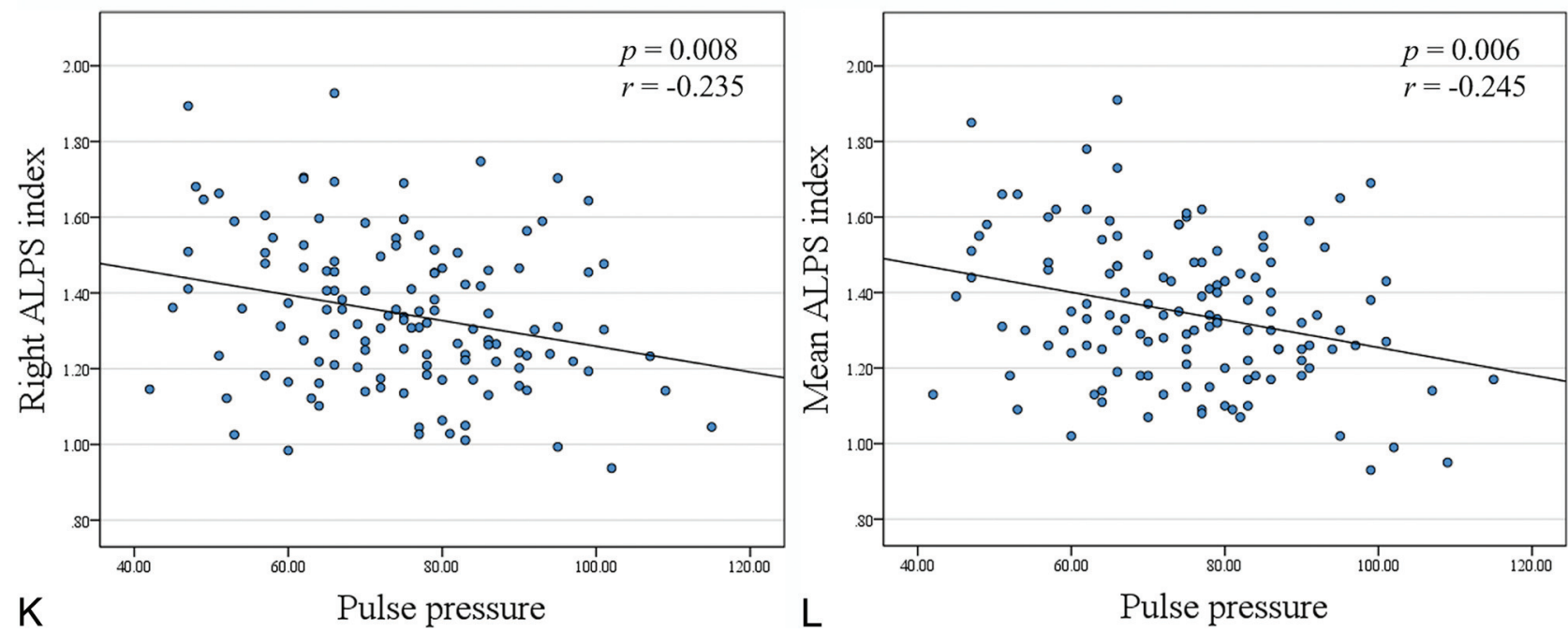

K

Pulse pressure

FIG 3. Continued

enters the brain along the cortical pial arteries by labeling the CSF via an injection of a fluorescent tracer into the cisterna magna CSF in mice. CSF dynamics have also been observed using intrathecal or intravenous injections of gadolinium-based contrast agents as tracers. ${ }^{3,32-39}$ However, the invasive injection of gadolinium-based contrast agents carries risks. Intrathecal administration of high-dose gadolinium-based contrast agents may cause gadolinium deposition in the globus pallidus and dentate nucleus ${ }^{40,41}$ or serious gadolinium encephalopathy, which includes nausea, dyspnea, subjective chills, delirium, dysarthria, 
spastic pain of the lower extremities, limb ataxia, and gaze-evoked nystagmus. ${ }^{42-44}$ Moreover, the tracking method requires several hours to track the distribution of a tracer in the brain, and monitoring glymphatic activity in real-time is challenging.

In contrast, the ALPS index is a noninvasive tool to assess the glymphatic system using DWI, which has a short acquisition time. As mentioned previously, the ALPS method is based on the hypothesis that diffusion plays an important role in fluid transport in the parenchyma. Fluid transport was believed to be caused mainly by cardiac pulsatility. ${ }^{13,29}$ However, Smith et $\mathrm{al}^{45}$ argued against this theory and suggested that diffusion played an important role in the transport of neurofluids. Several reports using mathematic modeling have further demonstrated the involvement of diffusion and advection in fluid transport in the parenchyma. ${ }^{46,47}$ Martinac and Bilston ${ }^{26}$ showed that diffusion is most likely the primary mechanism that drives fluid transport into the interstitial space. Taken together, diffusivity along the perivascular space could, at least in part, underlie glymphatic activity. Alterations of the glymphatic system in Alzheimer disease, ${ }^{15,48}$ idiopathic normal pressure hydrocephalus, ${ }^{18}$ diabetes, ${ }^{49}$ and Parkinson disease ${ }^{50}$ have been shown in living humans using the ALPS method, which is in line with the findings of studies using gadolinium-based contrast agents as tracers. $^{3,39,51}$ Additionally, Zhang et $\mathrm{al}^{52}$ recently reported that the ALPS index is significantly associated with the glymphatic clearance function calculated by glymphatic MR imaging, following intrathecal administration of gadolinium in patients with small-vessel disease $(r=-0.772$ to $-0.844, P<.001)$. The report strongly supported the clinical use of the ALPS index as a glymphatic biomarker.

This study has several limitations. First, it consisted of a relatively large number of participants compared with previous studies using the ALPS index; however, we obtained DWI data from only a single institution. Future research should include multisite data to evaluate the clinical utility of the ALPS index. In addition, given that a slight difference in the subject's head position can influence the ALPS index, we encourage investigations evaluating the influence of head motion on the calculation of the ALPS index. Second, we did not consider other physiologic statuses such as perfusion or pulsatile motion of the brain. Finally, Yokota et $\mathrm{al}^{18}$ showed that the size of the ROI may influence the calculated ALPS index, in which larger ROIs were more effective than smaller ROIs. Thus, further studies evaluating different sizes and shapes of ROIs for calculating the ALPS index are needed.

\section{CONCLUSIONS}

Our results are consistent with a model in which HT causes glymphatic dysfunction.

\section{ACKNOWLEDGMENTS}

We acknowledge Masahiro Abe for his help in data handling and graphic assistance. Additionally, the authors would like to thank Enago (www.enago.jp) for the English language review.

Disclosures: Junko Kikuta—RELATED: Grant: Japan Society for the Promotion of Science Grants-in-Aid for Scientific grant No. 20K16737.* Toshiaki TaokaUNRELATED: Grants/Grants Pending: Canon Medical Systems.* Akihiko Wada-
UNRELATED: Employment: Juntendo University. Yoshifumi Tamura—UNRELATED: Employment: Juntendo University. Hirotaka Watada_UNRELATED: Grants/Grants Pending: Novo Nordisk Pharma, Mitsubishi Tanabe Pharma, LifesScan Japan, Dainippon Sumitomo Pharma, Kyowa Kirin, Merck Sharp \& Dohme; Payment for Lectures Including Service on Speakers Bureaus: Mitsubishi Tanabe Pharma, Dainippon Sumitomo Pharma, Sanwa Kagaku, Takeda, Sanofi, Kowa Pharmaceuticals, Merck Sharp \& Dohme, Boehringer Ingelheim, Eli Lilly, Novo Nordisk Pharma. Shigeki Aoki—UNRELATED: Board Membership: Canon; Expert Testimony: Ono Pharmaceutical Co; Grants/Grants Pending: Medi-Physics, Daiich Sankyo, GE Healthcare, Eisai, FUJIFILM Toyama Chemical Co, Guerbet, Comments: grant for diagnostic radiology from Medi-Physics, Daiich Sankyo, GE Healthcare, Eisai, FUJIFILM Toyama Chemical Co, Guerbet; Payment for Lectures Including Service on Speakers Bureaus: Bayer, Canon, FUJIFILM Toyama Chemical Co, Eisai, Guerbet, GE Healthcare, Medi-Physics, Siemens, Daiichi Sankyo, Comments: payment for lectures/Chair from Bayer, Canon, FUJIFILM Toyama Chemical Co, Eisai, Guerbet, GE Healthcare, Medi-Physics, Siemens, Daiichi Sankyo. *Money paid to the institution.

\section{REFERENCES}

1. Iliff JJ, Wang M, Liao Y, et al. A paravascular pathway facilitates CSF flow through the brain parenchyma and the clearance of interstitial solutes, including amyloid beta. Sci Transl Med 2012;4:147ra111 CrossRef Medline

2. Jessen NA, Munk AS, Lundgaard I, et al. The glymphatic system: a beginner's guide. Neurochem Res 2015;40:2583-99 CrossRef Medline

3. Iliff JJ, Lee H, Yu M, et al. Brain-wide pathway for waste clearance captured by contrast-enhanced MRI. J Clin Invest 2013;123:12991309 CrossRef Medline

4. Louveau A, Smirnov I, Keyes TJ, et al. Structural and functional features of central nervous system lymphatic vessels. Nature 2015;523:337-41 CrossRef Medline

5. Faraco G, Park L, Zhou P, et al. Hypertension enhances $\mathbf{A} \beta$-induced neurovascular dysfunction, promotes $\beta$-secretase activity, and leads to amyloidogenic processing of APP. J Cereb Blood Flow Metab 2016;36:241-52 CrossRef Medline

6. Cifuentes D, Poittevin M, Dere E, et al. Hypertension accelerates the progression of Alzheimer-like pathology in a mouse model of the disease. Hypertension 2015;65:218-24 CrossRef Medline

7. Tadic M, Cuspidi C, Hering D. Hypertension and cognitive dysfunction in elderly: blood pressure management for this global burden. BMC Cardiovasc Disord 2016;16:208 CrossRef Medline

8. Kress BT, Iliff JJ, Xia M, et al. Impairment of paravascular clearance pathways in the aging brain. Ann Neurol 2014;76:845-61 CrossRef Medline

9. Plog BA, Dashnaw ML, Hitomi E, et al. Biomarkers of traumatic injury are transported from brain to blood via the glymphatic system. J Neurosci 2015;35:518-26 CrossRef Medline

10. Rasmussen MK, Mestre H, Nedergaard M. The glymphatic pathway in neurological disorders. Lancet Neurol 2018;17:1016-24 CrossRef Medline

11. Rusanen M, Kivipelto M, Levälahti E, et al. Heart diseases and longterm risk of dementia and Alzheimer's disease: a population-based CAIDE study. J Alzheimers Dis 2014;42:183-91 CrossRef Medline

12. Turana Y, Tengkawan J, Chia YC, et al. Hypertension and dementia: a comprehensive review from the HOPE Asia Network. J Clin Hypertens (Greenwich) 2019;21:1091-98 CrossRef Medline

13. Mestre $\mathrm{H}$, Tithof $\mathrm{J}$, Du T, et al. Flow of cerebrospinal fluid is driven by arterial pulsations and is reduced in hypertension. Nat Commun 2018;9:4878 CrossRef Medline

14. Mortensen KN, Sanggaard S, Mestre H, et al. Impaired glymphatic transport in spontaneously hypertensive rats. J Neurosci 2019;39:6365-77 CrossRef Medline

15. Taoka T, Masutani $Y$, Kawai $H$, et al. Evaluation of glymphatic system activity with the diffusion MR technique: diffusion tensor image analysis along the perivascular space (DTI-ALPS) in Alzheimer's disease cases. Jpn J Radiol 2017;35:172-78 CrossRef Medline

16. Someya Y, Tamura Y, Kaga H, et al. Skeletal muscle function and need for long-term care of urban elderly people in Japan (the 
Bunkyo Health Study): a prospective cohort study. BMJ Open 2019;9:e031584 CrossRef Medline

17. Jenkinson M, Beckmann CF, Behrens TE, et al. FSL. Neuroimage 2012;62:782-90 CrossRef Medline

18. Yokota H, Vijayasarathi A, Cekic M, et al. Diagnostic performance of glymphatic system evaluation using diffusion tensor imaging in idiopathic normal pressure hydrocephalus and mimickers. Curr Gerontol Geriatr Res 2019;2019:1-10 CrossRef Medline

19. Aronow WS. Hypertension and cognitive impairment. Ann Transl Med 2017;5:259 CrossRef Medline

20. Kario K, Pickering TG. Blood pressure variability in elderly patients. Lancet 2000;355:1645-46 CrossRef Medline

21. Adler S, Martinez J, Williams DS, et al. Positive association between blood brain barrier disruption and osmotically-induced demyelination. Mult Scler 2000;6:24-31 CrossRef Medline

22. Yamamoto $Y$, Ihara M, Tham C, et al. Neuropathological correlates of temporal pole white matter hyperintensities in CADASIL. Stroke 2009;40:2004-11 CrossRef Medline

23. Kamagata K, Andica C, Shimada K, et al. Effects of arterial stiffness on cerebral white matter integrity in the elderly. In: Proceedings of the Annual Meeting of the International Society for Magnetic Resonance in Medicine and the Society for MR Radiographers \& Technologists, Virtual; August 8-14, 2020

24. Joutel A, Corpechot C, Ducros A, et al. Notch3 mutations in CADASIL, a hereditary adult-onset condition causing stroke and dementia. Nature 1996;383:707-10 CrossRef Medline

25. Selwaness M, van den Bouwhuijsen Q, van Onkelen RS, et al. Atherosclerotic plaque in the left carotid artery is more vulnerable than in the right. Stroke 2014;45:3226-30 CrossRef Medline

26. Martinac AD, Bilston LE. Computational modelling of fluid and solute transport in the brain. Biomech Model Mechanobiol 2020;19:781800 CrossRef Medline

27. Bewick V, Cheek L, Ball J. Statistics review 7: correlation and regression. Crit Care 2003;7:451-59 CrossRef Medline

28. Krzesinski JM. Management of high blood pressure in peripheral arterial disease. Acta Chir Belg 2005;105:560-66 CrossRef Medline

29. Iliff JJ, Wang M, Zeppenfeld DM, et al. Cerebral arterial pulsation drives paravascular CSF-interstitial fluid exchange in the murine brain. J Neurosci 2013;33:18190-99 CrossRef Medline

30. Hadaczek P, Yamashita Y, Mirek H, et al. The "perivascular pump" driven by arterial pulsation is a powerful mechanism for the distribution of therapeutic molecules within the brain. Mol Ther 2006;14:69-78 CrossRef Medline

31. Braun M, IIif J. The impact of neurovascular, blood-brain barrier, and glymphatic dysfunction in neurodegenerative and metabolic diseases. Int Rev Neurobiol 2020;154C:413-36 CrossRef

32. Gaberel T, Gakuba C, Goulay R, et al. Impaired glymphatic perfusion after strokes revealed by contrast-enhanced MRI. Stroke 2014;45:3092-96 CrossRef Medline

33. Di Chiro G, Knop RH, Girton ME, et al. MR cisternography and myelography with Gd-DTPA in monkeys. Radiology 1985;157:37377 CrossRef Medline

34. Biceroglu H, Albayram S, Ogullar S, et al. Direct venous spinal reabsorption of cerebrospinal fluid: a new concept with serial magnetic resonance cisternography in rabbits. J Neurosurg Spine 2012;16:394401 CrossRef Medline

35. Naganawa S, Nakane T, Kawai H, et al. Gd-based contrast enhancement of the perivascular spaces in the basal ganglia. MRMS 2017;16:61-65 CrossRef
36. Naganawa S, Nakane T, Kawai $\mathrm{H}$, et al. Age dependence of gadolinium leakage from the cortical veins into the cerebrospinal fluid assessed with whole brain 3D-real inversion recovery MR imaging. Magn Reson Med Sci 2019;18:163-69 CrossRef

37. Naganawa $S$, Nakane T, Kawai $H$, et al. Lack of contrast enhancement in a giant perivascular space of the basal ganglion on delayed flair images: implications for the glymphatic system. Magn Reson Med Sci 2017;16:89-90 CrossRef Medline

38. Siebner HR, von Einsiedel HG, Conrad B. Magnetic resonance ventriculography with gadolinium DTPA: report of two cases. Neuroradiology 1997;39:418-22 CrossRef Medline

39. Ringstad G, Vatnehol SA, Eide PK. Glymphatic MRI in idiopathic normal pressure hydrocephalus. Brain 2017;140:2691-2705 CrossRef Medline

40. Kanda $\mathrm{T}$, Ishii $\mathrm{K}$, Kawaguchi $\mathrm{H}$, et al. High signal intensity in the dentate nucleus and globus pallidus on unenhanced T1-weighted MR images: relationship with increasing cumulative dose of a gadolinium-based contrast material. Radiology 2014;270:834-41 CrossRef

41. Taoka T, Naganawa S. Gadolinium-based contrast media, cerebrospinal fluid and the glymphatic system: possible mechanisms for the deposition of gadolinium in the brain. Magn Reson Med Sci 2018;17:111-19 CrossRef Medline

42. Samardzic D, Thamburaj K. Magnetic resonance characteristics and susceptibility weighted imaging of the brain in gadolinium encephalopathy. J Neuroimaging 2015;25:136-39 CrossRef Medline

43. Arlt S, Cepek L, Rustenbeck HH, et al. Gadolinium encephalopathy due to accidental intrathecal administration of gadopentetate dimeglumine. J Neurol 2007;254:810-12 CrossRef Medline

44. Provenzano DA, Pellis Z, DeRiggi L. Fatal gadolinium-induced encephalopathy following accidental intrathecal administration: a case report and a comprehensive evidence-based review. Reg Anesth Pain Med 2019;44:721-79 CrossRef

45. Smith AJ, Yao X, Dix JA, et al. Test of the 'glymphatic' hypothesis demonstrates diffusive and aquaporin-4-independent solute transport in rodent brain parenchyma. eLife 2017;6:e677679 CrossRef Medline

46. Asgari M, de Zélicourt D, Kurtcuoglu V. Glymphatic solute transport does not require bulk flow. Sci Rep 2016;6:38635 CrossRef Medline

47. Koundal S, Elkin R, Nadeem S, et al. Optimal mass transport with Lagrangian workflow reveals advective and diffusion driven solute transport in the glymphatic system. Sci Rep 2020;10:1990 CrossRef Medline

48. Steward CE, Venkatraman VK, Lui E, et al. Assessment of the DTIALPS parameter along the perivascular space in older adults at risk of dementia. J Neuroimaging 2021;31:569-78 CrossRef Medline

49. Yang G, Deng N, Liu Y, et al. Evaluation of glymphatic system using diffusion MR technique in T2DM cases. Front Hum Neurosci 2020;14:300 CrossRef Medline

50. Chen HL, Chen PC, $\mathrm{Lu} \mathrm{CH}$, et al. Associations among cognitive functions, plasma DNA, and diffusion tensor image along the perivascular space (DTI-ALPS) in patients with Parkinson's disease. Oxid Med Cell Longev 2021;2021:4034509 CrossRef Medline

51. Jiang Q, Zhang L, Ding G, et al. Impairment of the glymphatic system after diabetes. J Cereb Blood Flow Metab 2017;37:1326-37 CrossRef Medline

52. Zhang W, Zhou Y, Wang J, et al. Glymphatic clearance function in patients with cerebral small vessel disease. Neuroimage 2021;238:118257 CrossRef Medline 